\title{
Economics of Science in the Time of Data Economy and Gigabit Society
}

\author{
Michal Boni
}

In a period that Europe prepares to launch its new multi-annual programme for research and innovation it is important to step back and reflect on the broader social and economic benefits that Big Science and curiosity-driven research have brought to society and moreover how we can work together to maximize this impact. Further engaging citizens, offering more opportunities for young people, exploiting the tools presented by digital technologies are some of the challenges that I highlight/discuss in this essay.

The possibilities to build a real ecosystem of knowledge economy rely on many factors, which need to be seen together. It is the concept of the knowledge society development, the institutional and financial background for the Research and Innovation (R\&I) growth and the unbelievable data economy increase over the last years. All of them have contributed to the re-designed model of the knowledge economy. Science and innovation is now in the heart of the future development and is building competitive advantages of the European Union in the mid and long-term perspective.

In 2018, the innovation becomes the crucial reference point to establish one of the key growth engine for the EU under the next Multiannual Financial Framework. The proposed Horizon Europe for 2021-2027 was equipped by the European Commission with nearly EUR 100 billion. This was fully supported by the European Parliament, even if some wished to allocate more, ca. EUR 120 billion. ${ }^{1}$

\footnotetext{
${ }^{1}$ In the new document presented on May 2020 by the European Commission the position of the Horizon Europe is very strong, with the similar expenditures, and with some reorientations addressed to the new post-Covid challenges, As R\&I role in building the sustainable, inclusive and resilient recovery, in addition on virology, vaccines development, treatment and diagnostics, also acceleration of the twin digital and green transition (COM (2020) 442 final), The EU budget powering the recovery plan for Europe, May 2020.
}

M. Boni ( $\triangle)$

University of Social Science and Humanities (SWPS University), Warsaw, Poland

(C) The Author(s) 2021

H. P. Beck, P. Charitos (eds.), The Economics of Big Science, Science Policy

Reports, https://doi.org/10.1007/978-3-030-52391-6_3 
But it is not only financial envelope that matters. What is equally essential is the establishment of new rules on how to use the resources dedicated to the Science in practice.

In this respect, the fundamental issue was how to strengthen the cooperation between businesses and the science. What kind of conditions are necessary to develop innovative businesses, to inspire the new innovative spirit among scientists, to empower the collaborative forms helping both partners finding the most adequate and effective solutions. An improved partnership was a right solution, as it was oriented at various mutualities, supportive to achieve diverse advantages, but also to share burdens and concerns, as well as jointly solve problems. All of this with a strict requirement of the most appropriate and efficient leadership of business/scientific projects.

It does not mean, however, that only projects with a prospect of a success should be considered for partners' efforts. The magic of science very often means: unknown territory, unpredictability and yet the brave readiness to explore completely new areas. Hence, a certain 'right to fail' should be incorporated into the costs of the new system as it already exists within the start-ups.

This is the only way to go forward with innovative ideas, disruptive inventions and research and enhance the possibilities for commercialisation of the results of research. Future commercialisation must be at the birth of any research and innovation project. Over last years, in many research centres and universities the business/ science relations were understood as a new challenge and established as a brokering model, often in the form of special spinoffs.

Taking into account this critical juncture and considering the opportunity for the tipping point, I would like to describe a general framework needed for the Economics of Science, especially in the time of data economy and gigabit society.

The following requirements are necessary to be fulfilled.

Firstly, what kind of infrastructure is crucial for science development in the modern times?

The most expected and needed is well-designed data infrastructure. There are some scientifically oriented dimensions of the data infrastructure.

First of all, the network should be able to deliver a high quality transmission of data, especially Big Data with regard to certain areas, such as genetic sequences, biotechnology collections of data to mention but a few. As the high quality of infrastructure depends on the proper correlation between the speed and the latency, the most promising solution is the 5G infrastructure development in the European Union based - step by step — on the Giga frequencies. It requires substantial investments but they are indispensable for the future oriented data based science.

The next dimension relates to a very specific infrastructural challenge enabling appropriate data processing. The development and the accessibility of the SuperComputers with exascale possibilities will play crucial role in the coming years. Just as the open access to the quantum technologies makes the analytics much more timely and adjusted to the modern science. There is no possibility to develop data transmission and processing without the European Network of High Performance Computing Centres (EuroHPCinitiative). The "network" means that all processed data can flow and some works and tasks can be shifted and shared (in the framework 
of cooperation and shared responsibility) from one HPCC to another. The added value for the science is pivotal. Moreover, there are also economic efficiencies: the majority of the European HPC projects is generating financial returns; each euro invested on average provides in return EUR 867 in increased revenue/income and EUR 69 in profits. $^{2}$

The following dimension relies on proper design of the data infrastructure. It should be based on the European Data Strategy, which covers many areas and wasafter many years of debates-finally presented by the European Commission in February 2020. ${ }^{3}$ The proposed solution will allow establishing concrete Data Spaces with their functionalities: from agriculture, via industrial data, to the European Health Data Space. For the scientific field, the concept and implementation of the European Open Science Cloud was established a bit earlier, in 2017. However, for the effective functioning of all those Clouds and Spaces, a high level of the European interoperability (semantic) is required. It is essential for open and commonly addressed accessibility of data.

To sum up, the development of the data infrastructure is paramount for the economics of science as it can be one of the basic features of the European strategic autonomy. What is more, the digital components sovereignty with regard to the scientific research is necessary to ensure cybersecurity of all networks, including HPCC networks, SuperComputers and quantum technologies, and to provide for the necessary resilience against the digital scientific espionage.

Secondly, how can we use the European Open Science Cloud (EOSC) as an instrument to build and scale up the European Science?

Emblematically, the key word and practically, the key solution for the functionalities of the Science Cloud are based on the model of the openness. Presented by the European Commission, the triangle ${ }^{4}$ : open innovation-open science-open to the world, was crucial for the public debate. Essentially, it was focused on the open mind and on the open society concept. In that sense, open science addresses the characteristic features of the modern society, oriented at the expected and developing the knowledge and innovative society. It is clear that such concept was easier to implement before the time of populism and the increasing role of the post-truth era.

The open access trend, crucial for the digital game changer in many areas (industrial, scientific, related to the entertainment and all kinds of public services, also important for everyday life) is increasingly significant for the science. To have an access to research articles, to various scientific papers, using different types of the accessibility from subscription to the Creative Commons (and licenses related to this model), is basic for the dissemination of knowledge. What is more, access to the results of the studies and to the meta-data shared information about format and the

\footnotetext{
${ }^{2}$ Developing supercomputers in Europe, European Parliamentary Research Service, October 2017, p.3.

${ }^{3}$ European Commission, A European Strategy for data (COM(2020) 66 final), February 2020.

${ }^{4}$ Open Innovation, Open Science, Open to the World, May 2016, with introduction by Carlos Moedas, the Commissioner for the Science.
} 
content of the raw data, could further support the new scientific achievements. There is no possibility to be innovative without the broad access to many sources of information and knowledge.

But most importantly, the vehicle for the new science development is built on the significance of the Big Data, which at the same time is a fundament of the data driven economy.

Considerations around the Open Science Cloud model ought to take into account the importance of the rules for: data collecting, opening them for people (beneficiaries of the innovative solutions on one side and creators and scientists on the other), sharing, in some cases donating, processing, using, preserving and re-using, free flowing.

Also the context of the Open Science Cloud should be visible: The new legal framework for the free flow of data, harmonised implementation of the GDPR with a proper understanding of the meaning of "legitimate interest" and the use of "anonimisation" and "pseudonymization" schemes in the research, preparing for the future model of the ePrivacy directive, using new tools for the text and data mining under the Copyright Directive, openness for the accessibility of data for machine learning trainings, the re-use directive recast which is indispensable for genuine data exchange between Science and Research Centres (academia \& universities) in order to overcome differentiations and limits established by Member States.

The key drivers of the European Open Science Cloud development are:

- Data culture and the culture of the data sharing: European science must be anchored in a common culture of data stewardship, so that research data is recognised as a significant output of research and is appropriately curated throughout and after the period conducting the research. It also means-as I expressed above - the re-use model of research,

- FAIR data governance: means-Findable, Accessible, Interoperable, Reusable. It should be built upon inclusive stakeholders participation. The various kinds of policies and raising the awareness of these principles should go hand in hand with technical implementation and social infrastructure, including education and training. For all partners, such as university associations, research organisations, research libraries, research data repositories and others like educational brokers, new skills and competences are needed. They could help people to live among datasets and to manage them.

- Data management plans which seems to be obligatory in all research projects generating or collecting publicly funded research data. The meaningful part of those plans should be addressed to avoid data fragmentation and unequal access to quality information sets. It is linked to the fragmented access across scientific domains, countries and governance models, limited cross-disciplinary access to data sets, and non-interoperable services. The challenge is how to allow for universal access to the scientific data (as a common good?) and how to establish an equal level playing field for the EU researchers and all kinds of users. We must build the European Open Science Cloud as a one stop shop to find, access and use 
research data and services from multiple disciplines and platforms. Technically speaking, services and functionalities should be user driven.

It has taken three years to start legally and technically building the EOSC, and yet it has not been finalized. However, the developments are promising.

The problem is how to tie the European political will to make the research area much more open to fruitful exchanges and at the same time provide technical and infrastructural guarantee for effective-economically and scientificallydevelopment.

Thirdly, the data economy is only one side of the economics of science. The other is gigabit society. The society, which is involved in all digital game changer achievements and concerns. In this context, what is the role of the Science, especially with regard to societal and social expectations, needs, threats and fears?

The fundamental question is whether the science development or the economics of science require the participation of the society. And this participation should not only be seen with regard to the receipt of the research results (it is obvious and exists as an essential, conventional model), but also as a possibility to create innovative solutions together with the science and scientists and to explain many scientific and societal problems. In the broad sense: to explain the world.

The above mentioned is especially crucial for a transparent and open communication and the science literacy.

Especially at the time of populism, when populistic emotions and narratives use fake news and fake science and attacking the open minded societies as well as critical thinking patterns. Populistic prejudices and stereotypes undermine the evidence and knowledge based policies and solutions. The genuine science is really damaged by "science-like" discourse that uses emotion-biased propaganda.

New social and moral obligations emerge. We need to reconsider the following key social aspects of the functioning of the science, which will immensely impact the economics of the science and its effectiveness:

- How to communicate about science, publicly and understandably,

- What kind of literacies are needed to establish a new relationship between science and society - it should be the digital literacy, but complemented and enhanced by democratic, social, scientific, media and political literacy for better understanding the environment of the modern science and the science as an innovative game changer,

- How to tackle the problem of emotions in research, preparing scientists through relevant trainings, using the collective intelligence,

- How to develop citizens' engagement in science, how to support and develop the public engagement. Public engagement is an ongoing process. One of the key recommendations should be how to understand the public and do not regard it as a single homogeneous mass, but diverse audiences.

Different media audience often include citizens who are organised in communities with a certain interest or support for a particular scientific field. Are they ready to 
participate in all kinds of scientific processes? By adding the citizens' value to these processes and supporting the scientific efforts by participatory models.

The Citizens' Science is not the utopian dream.

It can be very important to give people the feeling of participation in the scientific process. For example this include data collection about the environmental threats by measuring the air pollution as a responsible community, complementary to research analysis or measuring the water pollution or its deficits. Such initiatives have a twofold role: On one hand, it can be an expression of the visible societal behaviour (to save the Planet), on the other it can be a serious contribution to the scientific analysis.

This is how the citizens and the society could emphasize their views on many issues and create the so-called demand for the science in general terms: to take into consideration, to start deeper analysis, to observe processes and to find conclusions and recommendations. It is the phenomenon of the usefulness of scientific efforts and studies. In my view, it is a meaningful complementary contribution to the innovative science: involving representatives of all generations, but especially numerous young creative minds (pupils from schools and students from universities) to shape the future and raise a common awareness of the development based on the real evidence and future proof orientations.

Paramount is to drive the science development ecosystem change with the involvement of the citizens. It is crucial for the positive results of the economics of science: societal approval, opportunities for participation, understandability, openness for all kinds of contexts.

There is one more meaningful context of this change: the AI development-as a General Purpose Technology, as a game changer for people' lives, for the economy and all industries and services. The challenge is whether humans, members of the Gigabit society, will keep the control over the unbelievable and fast developing technologies or lose it with all still unclear and unpredictable consequences. How to tackle the ethical problems related to the AI activities? How to support humans and develop their openness (to avoid the natural lack of the openness) for interactions with AI?

Citizens' view and a certain level of the oversight of the AI development by all involved stakeholders is necessary for making sure that the new scientific studies (based on AI) will be developed in agreement with humans needs.

Fourthly, considerations about the economics of science development lead us to the challenge of the excellence.

The best scientific results will be a lost opportunity without mobilising all resources to build the "ecosystem of excellence" along the entire value chain, starting from the research ideas and lab works, via creating the right incentives to accelerate the adoption of the solutions by all kinds of possible industries. Currently, and in the context of the AI development discussion, the "ecosystem of excellence"

\footnotetext{
${ }^{5}$ European Commission, White Paper. On Artificial Intelligence-A European approach to excellence and trust (COM(2020) 65 final), February 2020, part 1 Introduction.
} 
ought to cooperate with the "ecosystem of trust". 6 This creates a special added val coming from the human factor of the science development.

What is the specificity of the "ecosystem of excellence"?

In recent years, the European Union debated heavily on the innovation policy mix needed. It was clear that the research and development policies should be strongly linked with industrial and SME policies, education, skills and regional policies (with the concept of the smart specialisation). At the same time, some frameworks were necessary to adjust the financial support, state aid, tax policies and public procurement models. Additionally, it became obvious that the most adequate regulatory framework should function more effectively: single market and competition rules, all types of regulations: strong/strict and soft, standards and the schemes for certifications, and last but not least - the intellectual property rights and the transparent system of patents. In many discussions, the soft dimension of EU innovation policy mix was emphasised: partnerships and coordination initiatives, and the culture of innovation. $^{7}$

Some of those features are the key components of the supply side, some of them are crucial for the demand. This kind of view and understanding is significant for the economics of science providing for the market conditions for the science development.

Moreover, the "ecosystem of excellence" requires instruments, which could allow us to measure the effectiveness of the excellence in comparison to other science areas (the European Research Area in comparison to US, China, Japan, South Korea).

The following instruments can be considered: share of Science and Engineering publications in the top 1 citation percentile, general share of publications in global top $1 \%$ of cited publications, scientific excellence by fields, universities in Top 100 rankings, international mobility of researchers, shares of PhDs awarded and doctoral students in the US coming from EU, R\&D spending, patent activity indexes (especially for selected technologies in selected economies), patent applications for domestically owned inventions made abroad, successful international collaborations, etc. ${ }^{8}$

When looking at these instruments, the general conclusion is unfortunately full of doubts and concerns, which puts the objective of making Europe the Global Centre for Excellent Research under constraints.

If Europe wants to compete with leading powers at the global level, a proper scale of the science development is indispensable. It requires overcoming the current fragmented landscape of centres of competences for the research development and full support from the EU level and the Member States to the European Research

\footnotetext{
${ }^{6}$ Ibidem, part 4, An ecosystem of excellence.

${ }^{7}$ EU Innovation Policy_Part I, Building the EU innovation policy mix, European Parliamentary Research Service, In-depth analysis, p.7.

${ }^{8}$ indicated and mentioned in the: Europe-the Global Centre for Excellent Research, Study requested by the ITRE Committee of the European Parliament, Reinhilde Veugelers, Michael Baltensperger Policy Department for Economic, Scientific and Quality of Life Policies, April 2019.
} 
Area. It should be the most expected field and pattern of cooperation, taking all potentials from the scale into account. More synergies and networks between multiple European centres are necessary.

It must be established and supported by the European funding but it is critical how this funding is accessible. The size and scope of the funding should take into account the visible differences between some areas of Europe: the Eastern European countries are at the lower positions in all rankings, also with regard to the participation in the distribution of Horizon 2020 funded projects. ${ }^{9}$

It is clear, that the perspective of the economics of science could open not only the eyes of the policy makers in all European countries but also to wake the innovation potential of research centres in different countries (also lagging behind in terms of the science development) to pursue common collaborative partnerships. It should be done with some incentives, but also by showing the concrete advantages of building the common consortia. The concept of the common consortia means the participation of not only representatives from EU universities but also the representatives of the business from various parts of the EU.

The science/business partnership is a key condition for the commercialisation of the research works and is essential in the discussion on the economics of science. Additionally, it is clear that the above-mentioned consortia should have a businessoriented leadership. There is yet another, complementary reason to ensure the business oriented management: when analysing the potential of the European Science, we see-by looking at the number of citable documents ${ }^{10}$ by subjects-a competitive advantage of the EU Science in nearly all areas. The activities of the scientists and the results of the researches are excellent, but the commercial use of them is low and remains insufficient. This leads us to safely conclude that despite the excellent scientific potential we are not using it at the needed European scale.

Clearly, this observation also shows that excellence should be visible at all stages of the value chain of the economics of science-from initial ideas, via lab works, through pilot projects to the commercialised implementation.

The tipping point for the European economics of science is therefore clearusing all assets: from the mature model of the data infrastructure, via full adoption of the European Open Science Cloud, openness for citizens' participation in building the knowledge society with human factor (addressing the new technologies development), and the establishment of the "ecosystem of excellence".

It will lead us to the future: to the NEXT GENERATION of EUROPE, recovered and resilient.

\footnotetext{
${ }^{9}$ Ibidem, p.72 and 73.

${ }^{10}$ Ibidem, p.66.
} 
Open Access This chapter is licensed under the terms of the Creative Commons Attribution 4.0 International License (http://creativecommons.org/licenses/by/4.0/), which permits use, sharing, adaptation, distribution and reproduction in any medium or format, as long as you give appropriate credit to the original author(s) and the source, provide a link to the Creative Commons licence and indicate if changes were made.

The images or other third party material in this chapter are included in the chapter's Creative Commons licence, unless indicated otherwise in a credit line to the material. If material is not included in the chapter's Creative Commons licence and your intended use is not permitted by statutory regulation or exceeds the permitted use, you will need to obtain permission directly from the copyright holder. 